\title{
Estimation of the friction force during the needle insertion using the disturbance observer and the recursive least square
}

\author{
Yuta Fukushima* and Kiyoshi Naemura
}

\begin{abstract}
Needle insertion can be performed with the aid of an ultrasound (US) image. Detecting the position of a needle in a US image requires considerable technical expertise. To detect whether the needle has reached the target, the physician usually relies on physically sensing the amount of force being fed back from the tip of the needle to their fingertip. The goal of our research was to develop a force visualization system to assist physicians by providing them with a visual representation of the needle tip force. In this paper, we elucidated the characteristics of the force during the needle insertion, and designed the friction force estimation method. Needle tip force is difficult to estimate directly. The total insertion force acting on the needle was defined by adding the needle tip force to the friction force of the needle surface. Therefore, we proposed a method of estimating the change in the friction force by measuring the total insertion force during needle insertion using recursive least square method and a disturbance observer. The needle tip and friction forces were modeled on the basis of the results of in vitro experiments used pork back ribs. The experiments performed using the coaxial needle. This needle could measure the total insertion force and the needle tip force separately in real time. The validity of the designed estimator was evaluated by using the force values obtained by using a coaxial needle. As a result, the estimated friction force and measured friction force were found to be qualitatively consistent. However, a slight error was observed.
\end{abstract}

Keywords: Percutaneous therapy; Needle insertion; Modeling; Recursive least square; Disturbance observer

\section{Background}

Needle insertion is commonly used as a means of administering medication or anesthetics, performing biopsies, and aspirating fluids. Certain needle insertion procedures, such as percutaneous ethanol injection and radiofrequency ablation, are classified as types of minimally invasive surgery. If such procedures were performed without any image of the patient, however, human error may result in the insertion of the needle into an unintended target. Although needle insertion can be performed with the aid of an ultrasound (US) image, US images cannot be used for patients with large amounts of body fat. Furthermore, detecting the position of a needle in a US image requires considerable technical expertise. To detect whether the needle has reached the target, the physician usually relies

*Correspondence: d111200489@edu.teu.ac.jp

Tokyo University of Technology, Graduate School of Bionics Computer and Media Science, Katakuramachi, Hachioji City 192-0982, Tokyo, Japan on physically sensing the amount of force being fed back from the tip of the needle to their fingertip. Detection success thus varies according to the degree of proficiency of the individual. Thus, it would be possible to improve the insertion accuracy if a visual representation of the needle tip force could be provided to the physician. The current study focused on the regions where the needle tip force changes sharply upon reaching a target, such as the cava, blood vessels, and cancerous tumors.

The forces acting on the needle tip include those generated by cutting the tissue and the reactive force from the tissue [1]. Therefore, the needle tip force has been difficult to estimate simply by considering the total insertion force. Rather, the needle tip force should be obtained by subtracting the estimated friction force from the total insertion force.

There have been several recent studies on force visualization during needle insertion. Okamura et al. [1] 
and Simone et al. [2] elucidated, from an experiment using a cow liver, that the force acting on a needle can be modeled by summing the friction force, the cutting force, and the stiffness force. Kobayashi et al. [3] modeled the friction force based on the relative velocity between the needle and the tissue through an experiment using a hog liver. The purpose of these studies was not to detect the penetration of the needle into the tissue; rather, the estimated force was compared with the measured true value in real time. The friction force acting on the needle proved difficult to measure. Kataoka et al. [4] and Washio et al. [5] developed a coaxial needle consisting of an inner needle and outer sheath cannula. This needle could measure the total insertion force and the needle tip force separately in real time. The friction force could then be calculated by subtracting the measured needle tip force from the total insertion force.

The friction coefficient, stiffness, and damping coefficient of living tissue change in real time. Carra et al. [6] proposed a model in which the total insertion force was decomposed into a stiffness force, friction force, and cutting force. This model could explain the sharp decrease in the total insertion force. However, they did not perform an evaluation of the model using living tissue data. In a related study on the changes in the parameter estimates, Umeno et al. [7] proposed a method of estimating the air pressure in tires by using the recursive least square (RLS) method and a disturbance observer. This method was capable of estimating changing parameters in real time. RLS is an adaptive filter with a low calculation cost, and it is capable of estimating the coefficient quickly. Baurbe et al. $[8,9]$ used RLS to produce a highly accurate model of the total insertion force. They did not, however, develop a method of separating the needle tip force from the friction force. Moreover, the evaluation produced by the model did not use the needle tip force and friction force that were measured in real time.

The goal of our research was to develop a force visualization system to assist physicians by providing them with a visual representation of the needle tip force. In this paper, we elucidated the characteristics of the force during the needle insertion, and designed the friction force estimation method. Needle tip force is difficult to estimate directly. Estimation of the friction force has an important implication to estimate the needle tip force. To estimate the friction force, we proposed a method of estimating the change in the friction force by measuring the total insertion force during needle insertion using RLS and a disturbance observer. The estimated friction force using the proposed method allowed us to determine the estimation accuracy, but raised problems when compared with the measured true value obtained with a coaxial needle. The novelty of this study was to elucidate the characteristic of the force during and after needle insertion.

\section{Methods}

\section{Characteristics of the needle insertion force} Coaxial needle

Figure 1 shows a pattern diagram of the force acting on a needle during its insertion. The total insertion force $f_{\text {needle }}$ was defined by adding the needle tip force $f_{\text {tip }}$ to the friction force $f_{\text {friction }}$ of the needle surface [4]. The friction force changed with variations in the pressure applied to the needle by the tissue and the contact area between the needle and the tissue [10]. To measure the force acting on the needle during insertion, the coaxial needle was developed as shown in Figure 2. The coaxial needle consisted of a 19 gauge inner needle (Safelet cannula GA, Nipro Co., Japan) and a 16 gauge outer sheath cannula designed for this experiment. The force acting on the needle was measured by two force sensors. The needle tip force acting on the inner needle was measured by a load cell TL3B09-50 N, (Tec Gihan Co. Ltd., Japan), and the total insertion force was measured by another load cell (LMC-21023, Nissho electric works Co. Ltd., Japan). The rated capacity of the force sensors was $50 \mathrm{~N}$, and $100 \mathrm{~N}$, respectively. The friction force was obtained by subtracting the needle tip force from the total insertion force. The designed coaxial needle included the error $1 \mathrm{~N}$ that is derived from experiment equipment.

\section{Needle insertion experimental setups}

The experimental setups are shown in Figure 3. In the percutaneous needle insertion procedure, the needle penetrates skin, fat, and muscle as insertion targets. Pork back ribs were used, as their multiple fat and muscle layers made them appropriate for the study. As the needle insertion targets, we prepared samples that were $20 \mathrm{~mm}$ thick. To minimize the effect of deformation, the test pieces were clamped by an acrylic board, as shown in Figure 4. The acrylic board was then mounted on the three axis stage, as shown in Figure 3. The coaxial needle was set on the motorized single axis stage (SGSP 26-100,

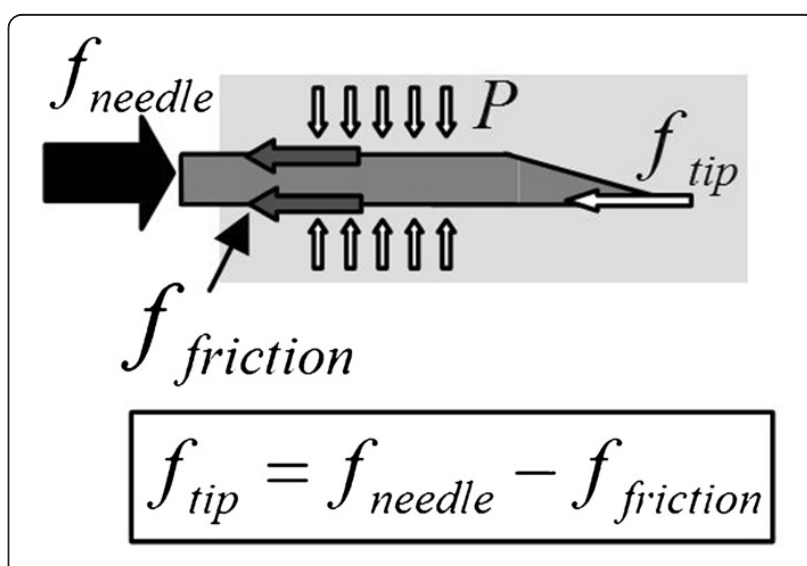

Figure 1 Force acting on the needle during the insertion. 


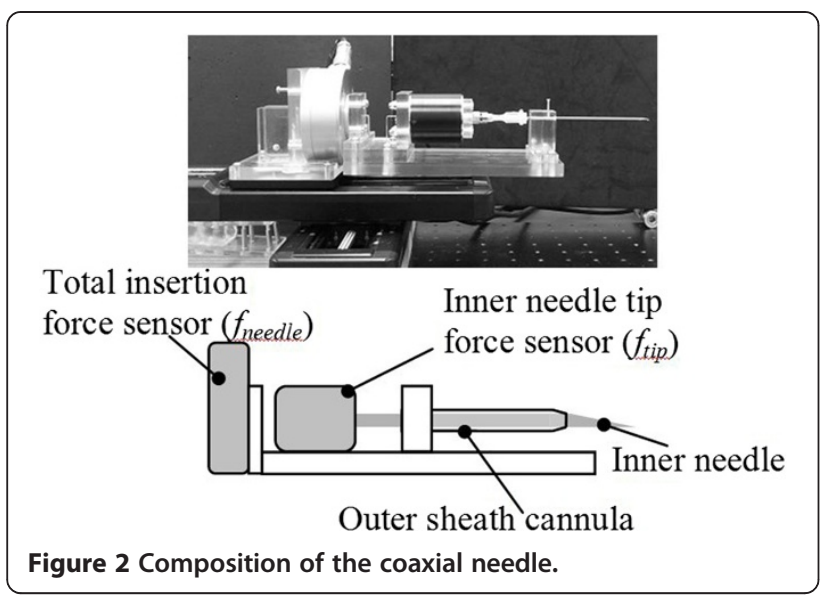

Sigma Koki Co. Ltd., Japan) and inserted into the test piece at a constant speed of $3.0 \mathrm{~mm} / \mathrm{s}$, in the same manner as in a former study [11]. The displacement was calculated from the velocity value set for the motorized single axis stage. The force acting on the needle was recorded by a data recorder at a cycle of $800 \mathrm{~Hz}$. Noise in the measured data was reduced by using the low pass filter of the Matlab/Simulink software. The needle was moved by $40 \mathrm{~mm}$, as shown in Figure 4, so as to puncture the test piece. The needle was first positioned on surface of the test piece, driven forward $40 \mathrm{~mm}$ until it emerged from the opposite side of the sample, and then stopped. The needle was inserted once into each test piece; nine insertions were performed.

\section{Experimental results}

Examples of the total insertion force, the friction force, and the needle tip force, as measured by the coaxial needle, are shown in Figure 5. All the forces reduced sharply and repeatedly. The insertion process involves three phases: phase I (when the needle is in contact with the test piece but has not yet formed an indentation), phase II (while the needle is being inserted into the test piece), and phase III (after the needle emerges from the opposite side of the test piece). These phases are shown in Figure 5 as I, II, and III, respectively.

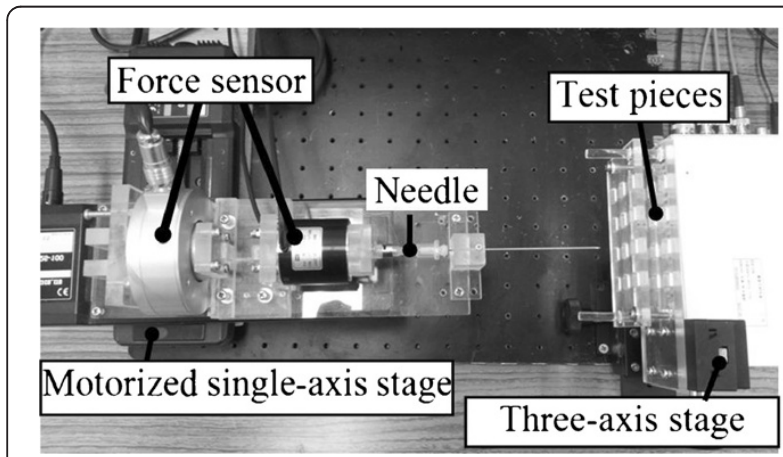

Figure 3 Needle insertion experimental setups.

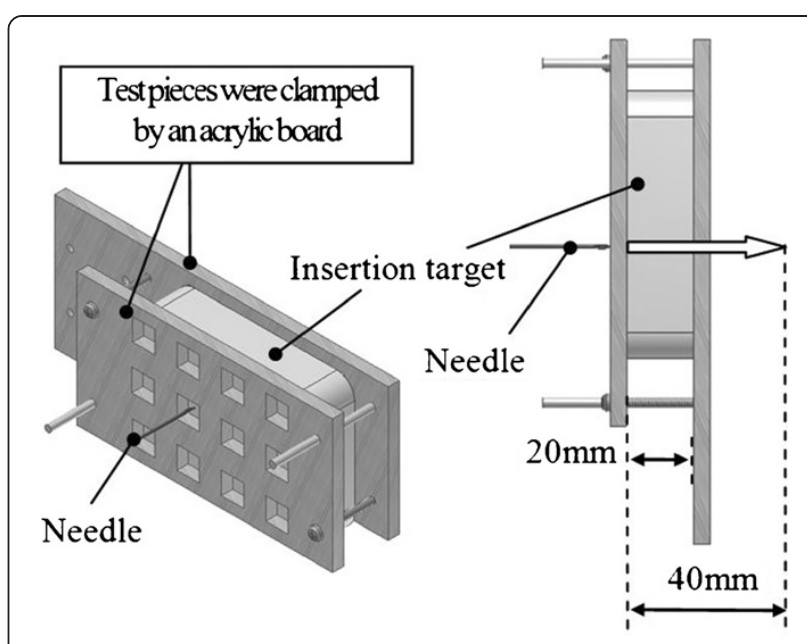

Figure 4 Explanation for preparing test pieces.

In phase $I$, the total insertion force increased non linearly until the needle penetrated the test piece. At that instant, the total insertion force was equivalent to the needle tip force, and the friction force was zero. Also, the test piece surface was deformed by about $5 \mathrm{~mm}$ by the needle.

In phase II, the total insertion force waveforms exhibited several peaks. On the other hand, the needle tip force remained constant at $0.5 \mathrm{~N}$ while the total insertion force increased. The total insertion force decreased with needle tip force.

An expanded graph of phase III is shown in Figure 6. After penetration by the needle, the needle tip force became zero, and the total insertion force and friction force decreased. After penetration, friction force was the only force acting on the needle. Then, the friction force increased slightly, as indicated by the arrow in Figure 6 .

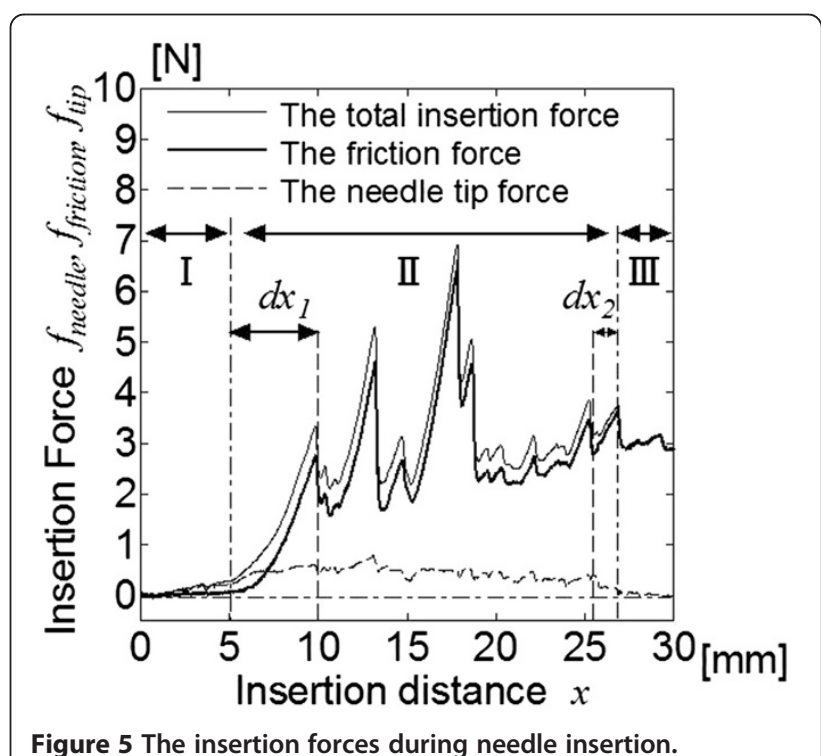




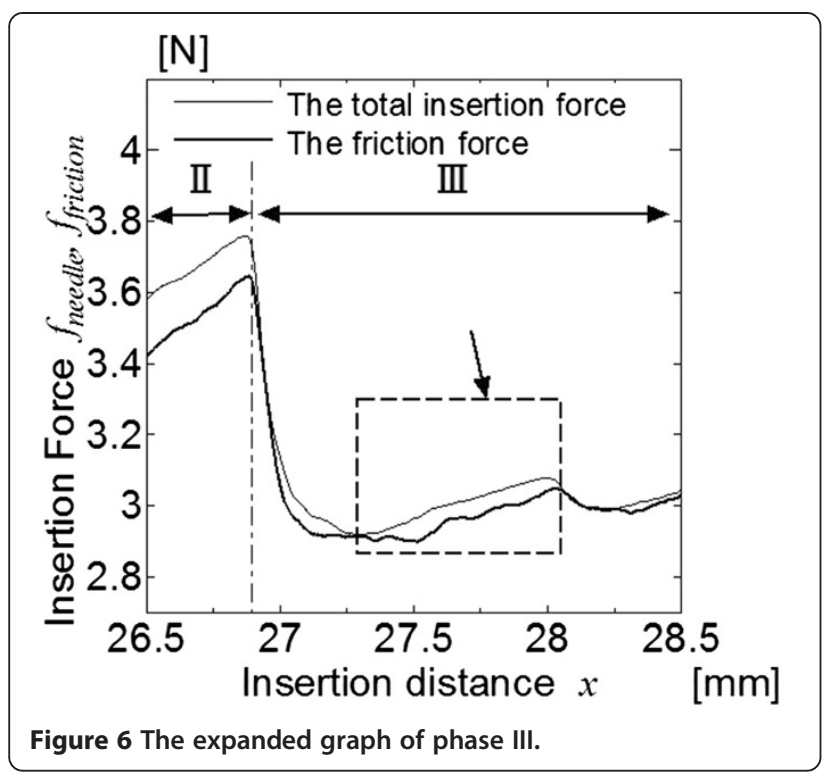

\section{Discussion}

In phase I, the total insertion force increased non linearly until the needle penetrated the tissue surface. Fung et al. [12] stated that the insertion force present until the instant at which the needle punctures the tissue surface was generated by the viscoelasticity of the tissue.

In phase II, the total insertion force, the friction force, and the needle tip force repeatedly increased and decreased. This can be explained by the fact that muscle tissue consists of fibers and bundles [13]. The needle insertion force increased while the muscle fibers deformed, and then fell as the muscular tissue ruptured. The needle tip force was a constant $0.5 \mathrm{~N}$ while the total insertion force increased.

In phase III, after the needle penetrated from the opposite side of the sample, the friction force increased slightly. This phenomenon was not observed in phase II, because the needle cut the thin tissue in the beginning of phase III. In cutting the thin tissue, the needle was deformed the tissue. After penetration of the tissue, the tissue moved to the opposite direction of the needle.

\section{Estimation of the friction force Model of the insertion force}

The needle insertion force should be modeled according to phase I, phase II, and phase III, as explained in the previous chapter, the detection of each phase proved difficult. Phase II was essential because it included the instant at which the needle penetrates from the opposite side of the sample. We elucidated that the dynamic friction force in single layer tissue presented as a product of the pressure applied to the needle by the tissue, the dynamic friction coefficient, and the area of the needle touching the tissue [10]. We assumed that the effect of viscosity was negligible in the current experiment. Heverly et al. [14] performed experiments to determine the velocity dependence of the needle tip force by using a porcine heart. We modeled the insertion force in single layer tissue of phase II as shown below.

$$
f_{\text {needle }}(x)=m \ddot{x}+s \dot{x}+\mu P d \pi x+\omega
$$

where

$$
\left\{\begin{array}{l}
f_{\text {friction }}(x)=\mu P d \pi x \\
f_{\text {tip }}(x)=s \dot{x}
\end{array}\right.
$$

Here, the mass of the needle was defined as $m$, the coefficient $\mu$ was the dynamic friction coefficient, the pressure $P$ was applied to the needle by the tissue, the diameter $d$ was the diameter of the needle, the vector $\omega$ was the disturbance vector which acted on the system, and the coefficient of the insertion speed $s$ was the coefficient that defined how the needle tip force changed with the insertion speed. Equation (1) explains is the force acting on the needle inserted to the silicone. If equation (1) applies to pork back ribs, the disturbance $\omega$ includes the parameter variation from preset value of the equation (1). Umeno et al. [7] presented the parameter variation estimation method using the RLS and disturbance observer as mentioned in introduction. In general the disturbance $\omega$ can estimate by the disturbance observer. The needle tip force was exhibited constant value, while the needle cut the tissue at the fixed speed as shown in chapter II. The variation value is only the friction force in equation (2) under fixed speed. Therefore, if the RLS and the disturbance observer were able to design, the friction force into pork back ribs under the constant insertion speed was able to estimate. In this paper, the disturbance observer was employed to estimate the disturbance $\omega$, and the variation parameter $\Delta \mu P$ in equation (1) was obtained using the RLS.

\section{Design of the disturbance observer}

Equation (1) was deformed to the linear time invariant system, as follows.

$$
\begin{aligned}
& \frac{d}{d t}\left[\begin{array}{l}
x \\
\dot{x}
\end{array}\right]=\left[\begin{array}{cc}
0 & 1 \\
-\mu P d \pi / m & -s / m
\end{array}\right]\left[\begin{array}{l}
x \\
\dot{x}
\end{array}\right]+\left[\begin{array}{c}
0 \\
1 / m
\end{array}\right] \lambda+\left[\begin{array}{c}
0 \\
-1 / m
\end{array}\right] \omega \\
& y=\left[\begin{array}{ll}
1 & 0
\end{array}\left[\begin{array}{l}
x \\
\dot{x}
\end{array}\right] .\right.
\end{aligned}
$$

Here, the input vector $\lambda$ was the total insertion force $f_{\text {needle. }} y$ was control output vector. The state variables were set as the insertion distance $x$ and the insertion speed $\dot{x}$. The state variable matrix $X$, the system coefficient matrix $A$, the driven matrix $B$, the output matrix $C$, and the coefficient matrix $D$ of the disturbance vector 
$\omega$ that was the determining matrix for the disturbance invasion route were defined as

$$
\begin{aligned}
X=\left[\begin{array}{l}
x \\
\dot{x}
\end{array}\right] & B=\left[\begin{array}{c}
0 \\
1 / m
\end{array}\right] \\
A=\left[\begin{array}{cc}
0 & 1 \\
-\mu \mathrm{Pd} \pi / m & -s / m
\end{array}\right]=\left[\begin{array}{ll}
A_{11} & A_{12} \\
A_{21} & A_{22}
\end{array}\right] & C=\left[\begin{array}{cc}
1 & 0
\end{array}\right] \\
D & =\left[\begin{array}{c}
0 \\
-1 / m
\end{array}\right]=\left[\begin{array}{l}
D_{11} \\
D_{21}
\end{array}\right] .
\end{aligned}
$$

Here, if the system changed, the disturbance $D \omega$ was included in the system noise and the fluctuation components. So, we designed the system to estimate $\omega$.

If the estimation speed of the observer was assumed to be sufficiently fast, equations (3) and (4) were deformed as extended systems expressing equations (6) and (7).

$$
\begin{aligned}
& \frac{d}{d t}\left[\begin{array}{l}
x \\
\dot{x} \\
\omega
\end{array}\right]=\left[\begin{array}{ccc}
A_{11} & A_{12} & D_{11} \\
A_{21} & A_{22} & D_{21} \\
0 & 0 & 0
\end{array}\right]\left[\begin{array}{c}
x \\
\dot{x} \\
\omega
\end{array}\right]+\left[\begin{array}{c}
0 \\
1 / m \\
0
\end{array}\right] \lambda . \\
& Y=\left[\begin{array}{lll}
1 & 0 & 0
\end{array}\right]\left[\begin{array}{c}
x \\
\dot{x} \\
\omega
\end{array}\right] .
\end{aligned}
$$

In the extended system, the state variable matrix $Z$, the system coefficient matrix $M$, the driven matrix $N$, and the output matrix $O$ were defined as

$$
\begin{gathered}
Z=\left[\begin{array}{c}
x \\
\dot{x} \\
\omega
\end{array}\right]=\left[\begin{array}{l}
X \\
\omega
\end{array}\right] \quad N=\left[\begin{array}{c}
0 \\
1 / m \\
0
\end{array}\right]=\left[\begin{array}{l}
B \\
0
\end{array}\right] \\
M=\left[\begin{array}{ccc}
A_{11} & A_{12} & D_{11} \\
A_{21} & A_{22} & D_{21} \\
0 & 0 & 0
\end{array}\right] \quad O=\left[\begin{array}{lll}
1 & 0 & 0
\end{array}\right]=\left[\begin{array}{ll}
C & 0
\end{array}\right] .
\end{gathered}
$$

Thus, we could design an observer to estimate the unmeasured state and the disturbance $\omega$.

There are several types of disturbance observer, such as a minimum order observer and a full order observer. In this study, we used a full order observer to compare the real state $(x$ and $\dot{x}$ ) to the estimated state $X$. The full order observer was designed as shown by equations (9) and (10).

$$
\begin{aligned}
& \dot{\hat{Z}}=M \hat{Z}+N \lambda+L(Y-O \hat{Z}) . \\
& \hat{Y}=O \hat{Z} .
\end{aligned}
$$

Here, the matrix $\hat{Z}$ was the estimated value of state $Z$, the coefficient $L$ was the observer gain, and the matrix $\hat{Y}$ was the estimated value of output equation $Y$.

\section{Design of parameter variation method using RLS}

A parameter variation method was designed by applying the results of the study by Umeno et al. [7]. The general state system was defined as shown below, including the input $\lambda$, white noise $d$, and the state variable $X$. The matrix $A$ and $B$ were the foregoing matrixes in equation (5).

$$
\dot{X}=A X+B \lambda+d .
$$

If the parameter matrices $A$ and $B$ fluctuate, the system after fluctuation was given as

$$
\begin{aligned}
& \dot{X}=A X+B \lambda+(\triangle A X+\Delta B \lambda+d) \\
& \dot{X}=A X+B \lambda+D \omega .
\end{aligned}
$$

Here, the disturbance $D \omega$ was defined as

$$
D \omega=\Delta A X+\Delta B \lambda+d .
$$

Thus, the parameter fluctuation and disturbance could be defined as being equivalent.

If the estimation speed of the observer could be assumed to be sufficiently fast, the disturbance $D \omega$ could be approximated as

$$
D \hat{\omega} \cong \triangle A X+\Delta B \lambda+d .
$$

In the proposed model, the matrix $\Delta B$ becomes constant at zero. Thus, the matrix $\Delta A$ and the state $X$ were defined, and equation (14) could be deformed as

$$
D \hat{\omega} \cong \triangle A X+d
$$

The RLS algorithm was applied to equation (15), and that was discredited. The RLS algorithm for estimating the parameter fluctuation was given by

$$
\left\{\begin{array}{l}
H(n)=\frac{1}{K}\left[H(n-1)-\frac{H(n-1) X(n) X^{T}(n) H(n-1)}{K+X^{T}(n) H(n-1) X(n)}\right] \\
\Delta \hat{A}(n)=\Delta \hat{A}(n-1)-\frac{H(n-1) X(n)}{K+X^{T}(n) H(n-1) X(n)} e(n)
\end{array} .\right.
$$

The $H(n)$ was covariance matrix [15]. The predictive error $e(n)$ was given by

$$
e(n)=X^{T}(n) \Delta \hat{A}(n-1)-D \omega(n) .
$$

Here, the matrix $\Delta \hat{A}$ was the changing estimated value of matrix $A, K$ was the forgetting coefficient, and $n$ was the sample data number.

The fluctuation value $\Delta A$ obtained from the proposed method was the fluctuation value of the system matrix A from the initial value in equation (5). So, the presented method can concurrently estimate the values of changing parameters $\mu, P$, and $s$ in equation (1). These coefficients defined the relationship between the friction force and the needle tip force. In chapter II, the needle tip force remained constant during the increase in the total insertion force. This meant that the parameter defining the relationship with the needle tip force variation could 
not be generated. Thus, in this study, we assumed that the parameter fluctuation related to the needle tip force was negligible, and that the fluctuating parameter could be attributed to the fluctuation of $P$ and $\mu$. Figure 7 is a block diagram of the above. The disturbance observer estimated the disturbance and the state variable. The estimated disturbance included the fluctuating parameters such as $\mu$ and $P$. The fluctuating parameters were estimated by RLS using the estimated disturbance. The initial setting parameter included a measurement error. The proposed method compensated for this measurement error.

\section{Simulation conditions}

We evaluated the estimated friction force and the measured friction force obtained with the coaxial needle. Simulation was performed using the Matlab/Simulink software. The initial parameter for the estimation method was set as shown in Table 1. Parameters obtained from a silicone rubber sample [10] were employed. To evaluate the robustness of the disturbance observer, the dynamic friction coefficient $\mu$ was varied to one tenth of the base value 6.63 . If the forgetting coefficient $K$ for RLS is set between 0.0 and1.0, the estimation system is insusceptible of the estimated pre value $\hat{\theta}(n-1)$, and is susceptible by noise. So, the forgetting coefficient $K$ was set to 0.80 . The pole of equation (3) was $\left[\begin{array}{lll}-1.09 & -89.3 & 0.00\end{array}\right]^{\mathrm{T}}$. The observer gain $L$ should be set negative value than pole of equation (3) on the complex plane. The observer gain $L$ was set to [ $5.90 \times$ $\left.10^{3} 1.14 \times 10^{7}-1.74 \times 10^{10}\right]^{\mathrm{T}}$. The pole of proposed method included observer was set to $\left[-2.0 \times 10^{3}-2.0 \times 10^{3}-2.0 \times 10^{3}\right]$.

\section{Results}

\section{Estimation results}

The results of the simulation are shown in Figure 8. The calculated needle tip force was obtained by subtracting the estimated friction force from the measured total insertion force. The estimation accuracy of the needle tip

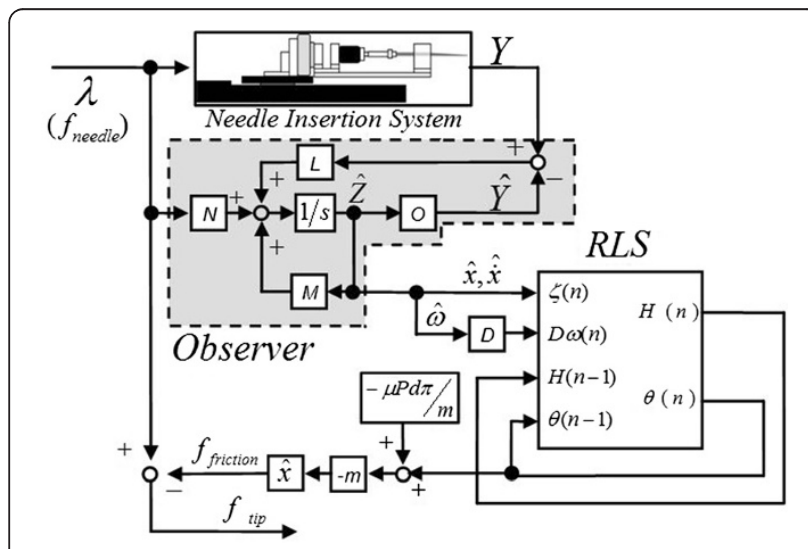

Figure 7 The block diagram of proposed method.
Table 1 Initial value for the friction force estimation method during the needle insertion

\begin{tabular}{lll}
\hline Needle diameter $d[\mathrm{~m}]$ & $1.60 \times 10^{-3}$ & \\
Needle mass $m[\mathrm{~kg}]$ & $2.18 \times 10^{-3}$ & \\
Dynamic friction coefficient $\mu$ & 0.663 & 6.63 \\
The Pressure of tissue to the needle $P\left[\mathrm{~N} / \mathrm{m}^{2}\right]$ & $63.7 \times 10^{2}$ & \\
The coefficient of velocity $s$ & 197 \\
\hline
\end{tabular}

force was determined by the estimated friction force. So, discussing the estimation accuracy of the needle tip force was identical with that of the estimated friction force. The three phases of needle insertion, as shown in Figure 5, are indicated by I, II, and III. In Figure 8, the friction force was qualitatively consistent, although a degree of error was observed. We defined the estimation error by subtracting the estimated friction force from the measured friction forces. The estimation error included the error $1 \mathrm{~N}$ that is derived from experiment equipment. The root mean square error (RMSE) was defined by

$$
R M S E=\sqrt{\frac{1}{b} \sum_{i=1}^{b}\left(\hat{F}_{f i}-F_{f i}\right)^{2}}
$$

Here, the integer number $b$ was the total number of the data, the data number was defined as $i$, the estimation value of the friction force was defined as $\hat{F}_{f i}$, and the measurement value of the friction force by the coaxial needle was defined as $F_{f i}$. The estimation error and the total insertion force were shown in Figure 9. The average error, the maximum error and the RMSE in each phase were shown in Table 2 and Table 3 respectively. Here, to

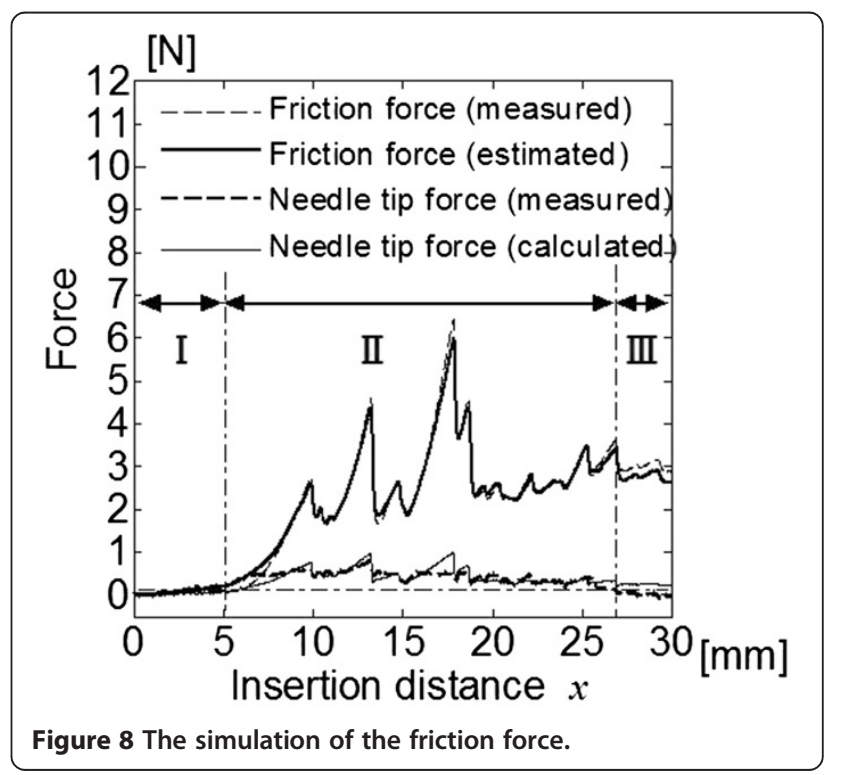




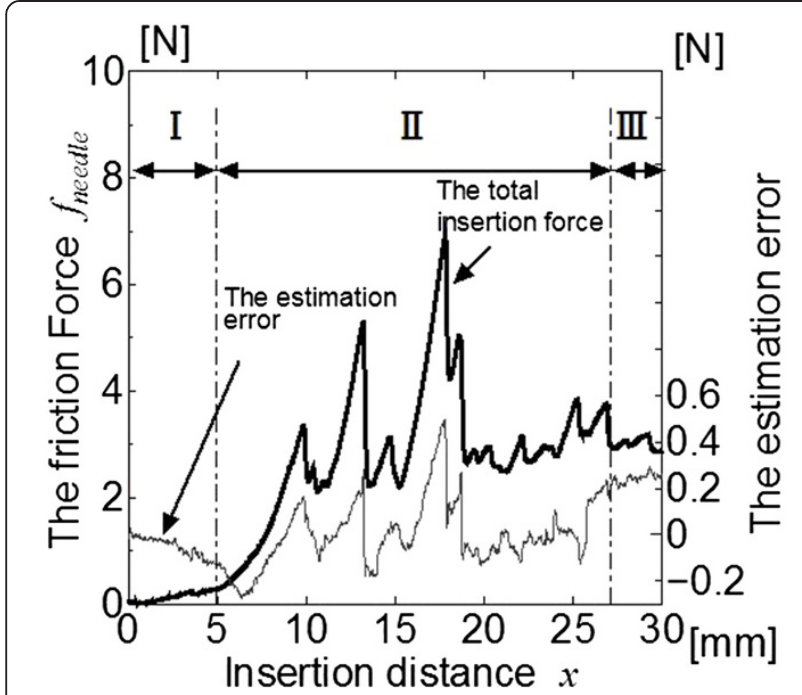

Figure 9 The estimation error from the friction force simulation ( $\mu$ : base value 6.63 ).

obtain the RSL of each phase, the integer numbers $b$ of each phase were set 1667 ( $x$ : 0.00 to $5.00 \mathrm{~mm}$ ), 7300 ( $x$ : 5.00 to $26.9 \mathrm{~mm}$ ), and $1034(x: 26.9$ to $30.0 \mathrm{~mm})$, respectively. The maximum estimation error in phase II was $-0.27 \mathrm{~N}$. The average estimation error was $-0.10 \mathrm{~N}$. The estimation error increased in the negative direction immediately after the total insertion force fell sharply in phase II. The RMSE were increased in both phase II and phase III.

The estimation error which $\mu$ was set to one tenth of the base value is shown in Figure 10. The result for the estimation error was completely consistent with the results for the estimation error by the base value. The RMSE which $\mu$ was set to one tenth of the base value is shown in Table 3 . The RMSE was also completely consistent with the result of the base value. Therefore, we found that changing the dynamic friction coefficient had no effect on the robustness of the disturbance observer.

\section{Discussion}

\section{Discussion}

The estimation error increased in phase I as shown in Figure 9. Phase I exhibited a characteristic in that the friction force did not act on the needle, although the proposed method could not capture the characteristic of the friction force in phase I. Thus, the larger estimation error was observed.

Table 2 The estimation error in each insertion phase

\begin{tabular}{lll}
\hline Phase & Average value $[\mathbf{N}]$ & Maximum error $[\mathbf{N}]$ \\
\hline $\mid$ & -0.05 & -0.13 \\
$\|$ & -0.10 & -0.27 \\
\|\| & 0.24 & 0.29 \\
\hline
\end{tabular}

Table 3 The RMSE in each insertion phase

\begin{tabular}{lll}
\hline Phase & RMSE [N] & \\
\cline { 2 - 3 } & $\boldsymbol{\mu}: \mathbf{6 . 6 3}$ & $\boldsymbol{\mu}: 6.63 \times \mathbf{1 / 1 0}$ \\
\hline I & 0.07 & 0.07 \\
$\|$ & 0.14 & 0.14 \\
$\mid I$ & 0.24 & 0.24 \\
\hline
\end{tabular}

In phase II, the total insertion force fell sharply as the muscle bundle was cut. The estimation error increased as the muscle bundle deformed, because the proposed method did not consider the characteristics of tissue deformation.

The friction force increased as a result of tissue relaxation, the estimation error could be observed in phase III. We assumed that the estimation error increased as a result of the tissue deformation, because the estimation error increased in the negative immediately after the total insertion force decreased sharply. Thus, the estimation error increased in insertion phases II and III, especially given that these phases included tissue cutting. The RMSE, indicating the dispersion from the true value, also increased in phases II and III. This means that the RMSE increases as a result of tissue cutting.

Changing the dynamic friction coefficient had no effect on the robustness of the disturbance observer. Thus, the proposed method was robust to difference of initial parameter. This means that the velocity estimated by the disturbance observer was greater than the parameter changing velocity. If the observer gain changed, so too did the estimation accuracy.

In the future, we will enhance the proposed method by including the effect of tissue deformation and relaxation.

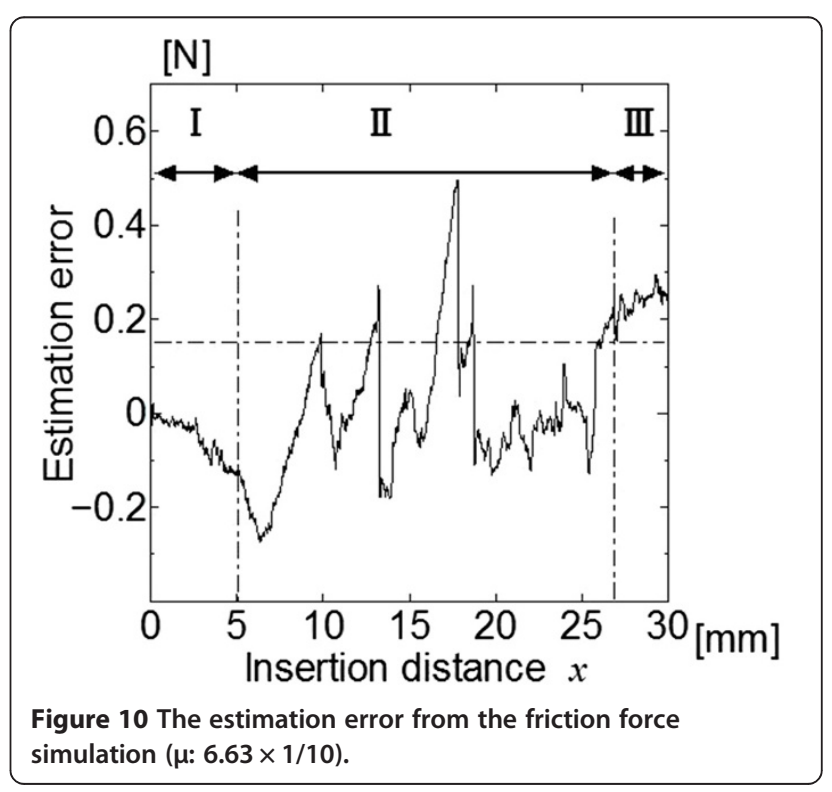




\section{Conclusion}

The goal of our research was to develop a force visualization system to assist physicians by displaying the needle tip force. In this paper, the needle tip and friction forces were modeled on the basis of the results of our experiments. The validity of the designed estimator was evaluated by using the force values obtained by using a coaxial needle. As a result, both forces were found to be qualitatively consistent. However, a slight error was observed. In the future, to reduce this estimation error, we will design a system that considers the force resulting from tissue deformation and characteristics of the insertion force.

\section{Competing interests}

The authors declare that they have no competing interests.

\section{Authors' contributions}

YF performed the needle insertion experiments, analyzed measured data, and drafted the manuscript. KN discussed results with YF and helped to draft the manuscript. All authors have read and approved the final manuscript.

\section{Acknowledgment}

The authors are grateful to Mr. H. Saito, Mr. T. Yamazaki and Mr. S. Nagatomo (UNISIS Corporation Japan) for their assistance in creating the outer sheath cannula. The authors also thank Dr. Y. Koseki of the National Institute of Advanced Industrial Science and Technology (AIST) for his advice on the coaxial needle.

Received: 14 January 2014 Accepted: 24 July 2014

Published online: 05 September 2014

\section{References}

1. Okamura AM, Simone C (2004) Force modeling for needle insertion into soft tissue. IEEE 51(10):1707-1716

2. Simone C, Okamura AM (2002) Modeling of Needle Insertion Forces for Robot-Assisted Percutaneous Therapy. IEEE international conference on robotics \& automation, Washington, DC, USA

3. Kobayashi Y, Sato T, Fujie MG (2009) Modeling of Friction Force based on Relative Velocity between Liver Tissue and Needle for Needle Insertion Simulation. IEEE International conference on engineering in medicine and biology society, Hilton Minneapolis, Minnesota, USA

4. Kataoka H, Washio T, Chinzei K, Mizuhara K, Simone C, Okamura AM (2002) Measurement of the Tip and Friction Force Acting on a Needle during Penetration. In: 5th international conference on medical image computing and computer assisted intervention, vol 2488, Lecture Notes in Computer Science. Springer, Tokyo, Japan, pp 216-223

5. Washio T, Chinzei K (2004) Needle Force Sensor, Robust and Sensitive Detection of the Instant of Needle Puncuture. 7th international conference medical image computing and computer assisted intervention, Saint Malo, Flance

6. Carra A, Avila-Vilchis JC (2010) Mutilayer Needle Insertion Modeling for Robotic Percutaneous Therapy. 4th international conference on bioinformatics and biomedical engineering, Chengdu, China

7. Umeno T (2009) Parameter estimation by using disturbance observer. J Japan Fluid Power Syst Soc 40(3):169-173

8. Barbe L, Bayle B, Mathelin MD, Gangi A (2007) Needle insertion modeling: Identifiability and limitations. In: David F (ed) Proceedings of IFAC, France

9. Barbe L, Bayle B, Mathelin MD, Gangi A (2009) In vivo model estimation and haptic characterization of needle insertion. Int J Robot Res 26(11):1154-1168

10. Fukushima Y, Saito K, Naemura K (2013) Estimation of the Cutting Force Using the Dynamic friction coefficient obtained by reaction force during the needle insertion. In: Mamoru M, Paolo B (eds) Proceedings of 1st CIRP international conference on BioManufacturing, Tokyo

11. Dimaio SP, Salcudean SE (2003) Needle insertion modeling and simulation. IEEE 19(5):864-875.
12. Fung YC (1993) Biomechanics: Mechanical Properties of Living Tissues. Springer, Berlin

13. Ushiki T (1989) Basic histology. Nankodo, Japan

14. Heverly M, Pierre D, Triedman J (2005) Trajectory Optimization for Dynamic Needle Insertion. IEEE International Conference on Robotics and Automation, Barcelona, Spain, 18-22 April 2005

15. Adachi S (2009) Basic of system identification. Tokyo Denki University Press, Japan

doi:10.1186/s40648-014-0014-7

Cite this article as: Fukushima and Naemura: Estimation of the friction force during the needle insertion using the disturbance observer and the recursive least square. $R O B O M E C H$ Journal 2014 1:14.

\section{Submit your manuscript to a SpringerOpen ${ }^{\odot}$ journal and benefit from:}

- Convenient online submission

- Rigorous peer review

- Immediate publication on acceptance

- Open access: articles freely available online

- High visibility within the field

- Retaining the copyright to your article

Submit your next manuscript at $>$ springeropen.com 